\title{
RESEARCH ANNOUNCEMENT
} \author{
APPEARED IN BULLETIN OF THE
AMERICAN MATHEMATICAL SOCIETY \\ AMERICAN MATHEMATICAL SOCIETY
Volume 27, Number 2, October 1992, Pages 239-242

\section{SMOOTH STATIC SOLUTIONS OF THE EINSTEIN-YANG/MILLS EQUATION}

\author{
J. Smoller, A. Wasserman, S. T. Yau, and B. McLeod
}

\begin{abstract}
We consider the Einstein/Yang-Mills equations in $3+1$ space time dimensions with $\mathrm{SU}(2)$ gauge group and prove rigorously the existence of a globally defined smooth static solution. We show that the associated Einstein metric is asymptotically flat and the total mass is finite. Thus, for non-abelian gauge fields the Yang/Mills repulsive force can balance the gravitational attractive force and prevent the formation of singularities in spacetime.
\end{abstract}

1

The only static, i.e., time independent, solution to the vacuum Einstein equations for the gravitational field $R_{i j}-\frac{1}{2} R g_{i j}=0$ is the celebrated Schwarzschild metric that is singular at $r=0$ [1]. Despite this defect, this solution has applicability for large $r$ to physical problems, e.g., the perihelion shift of Mercury. Similarly, the Yang/Mills equations $d^{*} F=0$, which unify electromagnetic and nuclear forces, have no static regular solutions on $\mathbb{R}^{4}[3]$. Furthermore, if one couples Einstein's equations to Maxwell's equations, to unify gravity and electromagnetism

$$
R_{i j}-\frac{1}{2} R g_{i j}=\sigma T_{i j}, \quad d^{*} F=0
$$

( $T_{i j}$ is the stress-energy tensor relative to the electromagnetic field $F_{i j}$ ), the only static solution is the Reissner-Nordström metric, which is again singular at the origin [1]. Finally, the Einstein-Yang/Mills (EYM) equations, which unify gravitational and nuclear forces, were shown in [4] to have no static regular solutions in $(2+1)$ space time dimensions for any gauge group $G$. We announce here that the contrary holds in $(3+1)$ space-time dimensions. Indeed, with $\mathrm{SU}(2)$ gauge group

1991 Mathematics Subject Classification. Primary 83C05, 83C15, 83C75, 83F05, 35Q75.

Received by the editors November 5, 1991 and, in revised form, January 29, 1992

The first author's research was supported in part by NSF Contract No. DMS-89-05205 and, with the second author, in part by ONR Contract No. DOD-C-N-00014-88-K-0082; the third author was supported in part by DOE Grant No. DE-FG02-88ER25065; the fourth author was supported in part by the U.K. Science and Engineering Council. 
(i.e., the weak nuclear force) we prove that the EYM equations (c.f. (1), where now $F_{i j}$ is the su(2)-valued Yang/Mills field), admit nonsingular static solutions, whose metric is asymptotically flat, i.e., Minkowskian. (Strong numerical evidence for this conclusion was obtained by Bartnik and McKinnon [2] who also derived the relevant equations.) Thus for non-abelian gauge fields, the Yang-Mills repulsive force can balance gravitational attraction and prevent the formation of singularities in spacetime. Viewed differently from a mathematical perspective, it is the nonlinearity of the corresponding Yang/Mills equations that allows the existence of smooth solutions.

The EYM equations are obtained by minimizing the action

$$
\int\left(-R+|F|^{2}\right) \sqrt{g} d x
$$

over all metrics $g_{i j}$ having signature $(-,+,+,+)$. These equations become

$$
R_{i j}=2 F_{i k} F_{j}^{k}-\frac{1}{2}|F|^{2} g_{i j} .
$$

Here $R$ is the scalar curvature associated to the metric $g_{i j}$ and $F$ is the Yang-Mills curvature. These formidable equations become more tractible if we consider static symmetric solutions.

\section{2}

The problem of finding static, symmetric nonsingular solutions of the EYM equations with $\mathrm{SU}(2)$ gauge group can be reduced to the study of the following system of ordinary differential equations

$$
\begin{gathered}
r^{2} A w^{\prime \prime}+\Phi w^{\prime}+w\left(1-w^{2}\right)=0 \\
r A^{\prime}+\left(2 w^{2}+1\right)=1-\frac{\left(1-w^{2}\right)^{2}}{r^{2}}, \\
2 r a T^{\prime}+\left(2 w^{2} A+\Phi / r\right) T=0 .
\end{gathered}
$$

Here $\Phi(r)=r(1-A)-\frac{\left(1-w^{2}\right)^{2}}{r}, A$ and $T$ are the unknown metric coefficients, $d s^{2}=-T^{-2}(r) d t^{2}+A^{-1}(r) d r^{2}+r^{2}\left(d \theta^{2}+\sin ^{2} \theta d \phi^{2}\right)$, and $w$ is the "connection coefficient" relative to the sought-for connection $\alpha=w \tau_{1} d \theta+\left[\cos \theta \tau_{3}+w \sin \theta \tau_{2}\right] d \phi$, $\tau_{1}, \tau_{2}$, and $\tau_{3}$ being the generators of the Lie algebra su(2). The associated curvature $F-d \alpha+\alpha \wedge \alpha$ is

$$
F=w^{\prime} \tau_{1} d r \wedge d \theta+w^{\prime} \tau_{2} d r \wedge(\sin \theta d \phi)-\left(1-w^{2}\right) \tau_{3} d \theta \wedge(\sin \theta d \phi) .
$$

If $<\tau_{i}, \tau_{j}>=-2 t r \tau_{i} \tau_{j}$ denotes the Killing form on su(2), and if $|F|^{2}=g^{i j} g^{k l} F_{i j} F_{k l}$, then an easy calculation gives

$$
|F|^{2}=2 w^{\prime} / r^{2}+\left(1-w^{2}\right)^{2} / r^{4} .
$$


FIGURE 1

In order that our solution has finite mass, i.e., that $\lim _{r \rightarrow \infty} r(1-A(r))<\infty$ we require that

$$
\lim _{r \rightarrow \infty}\left(w(r), w^{\prime}(r)\right) \text { be finite. }
$$

Furthermore, asymptotic flatness of the metric means that

$$
\lim _{r \rightarrow \infty}(A(r), T(r))=(1,1) .
$$

Finally, the conditions needed to ensure that our solution is nonsingular at $r=0$ are

$$
w(0)=1, \quad w^{\prime}(0)=0, \quad A(0)=1, \quad T^{\prime}(0)=0 .
$$

One sees from (2) that the first two equations do not involve $T$. Thus we first solve these for $A$ and $w$, subject to the above initial and asymptotic conditions.

\section{3}

We prove that under the above boundary conditions, every solution is uniquely determined by $w^{\prime \prime}(0) ; w^{\prime \prime}(0)=-\lambda$ is a free parameter. We seek a $\lambda>0$ such that there exists an orbit $\left(w(r, \lambda), w^{\prime}(r, \lambda)\right)$ that "connects two rest points." It is then not very difficult to prove that (4) will also hold.

A major difficulty is to show that the equations (2a), (2b) actually define a nonsingular orbit; i.e., that $w^{\prime}(r, \lambda)$ is bounded and that $A(r, \lambda)$ remains positive. Our first result is

Theorem 1. If $0 \leq \lambda \leq 1$, then in the region

$$
\Gamma=\left\{w^{2} \leq 1, w^{\prime} \leq 0\right\},
$$

$A(r, \lambda)>0$ and $w^{\prime}(r, \lambda)$ is bounded from below.

On the other hand, we can also prove (see Figure 1)

Theorem 2. If $\lambda>2$, then the solution of equations (2a), (2b), with initial conditions (5) blows up in $\Gamma$; i.e., $w^{\prime}(r)$ is unbounded.

If $\lambda$ is near zero, then by rescaling we can show that the orbit $\left(w(r, \lambda), w^{\prime}(r, \lambda)\right)$ exits $\Gamma$ through the line $w=-1$. Furthermore, for $\lambda=1$, numerical approximations 
indicate that $w^{\prime}$ becomes positive in the region $-1<w<0$. If this could be established rigorously, we could assert the existence of some $\bar{\lambda}, 0<\bar{\lambda}<1$, for which the corresponding orbit stays in $\Gamma$ for all $r \geq 0$, thereby proving (3). It would then be possible to prove that

$$
\left.\lim _{r \rightarrow \infty}(w(r, \bar{\lambda})), w^{\prime}(r, \bar{\lambda})\right)=(-1,0)
$$

and as a consequence, that (4) would also hold.

We can give a completely rigorous proof of the existence of a connecting orbit with $\lambda<2$, which we now outline. First Theorem 2 and the fact that for $\lambda$ near 0 the corresponding orbit exits $\Gamma$ through the line $w=-1$ implies that there is a smallest $\lambda=\bar{\lambda}$ for which the orbit $\left(w(r, \bar{\lambda}), w^{\prime}(r, \bar{\lambda})\right)$ does not exit $\Gamma$ through this line. Thus only the following two possibilities can arise:

$\left(\mathrm{P}_{1}\right)$ There is a real number $\bar{r}>0$ such that either $(\mathrm{a}) w^{\prime}(\bar{r}, \bar{\lambda})=0$, or (b) $A(\bar{r}, \bar{\lambda})=0$, or $(\mathrm{c}) w^{\prime}(r, \lambda)$ is unbounded for $r$ near $\bar{r}$.

$\left(\mathrm{P}_{2}\right)$ For all $r>0, w(r, \bar{\lambda})>-1, w^{\prime}(r, \bar{\lambda})<0$, and $A(r, \bar{\lambda})>0$.

In the case that $\left(\mathrm{P}_{2}\right)$ holds, we can show, as above, that both (6) and (7) hold. In order to rule out possibility $\left(\mathrm{P}_{1}\right)$, we consider several cases. The crucial case occurs when $A(\bar{r}, \bar{\lambda})=0, w^{\prime}(r, \bar{\lambda})$ is unbounded near $r=\bar{r}$, and $\Phi(\bar{r}, \bar{\lambda})=0$. Now set $\bar{w}=\lim _{r} \bar{r}_{r} w(r, \bar{\lambda})$. If $\bar{w}<0$, then defining $v(r, \lambda)=\left(A w^{\prime}\right)(r, \lambda)$, we show that $v$ satisfies a first order ode, and we can prove that for $\lambda<\bar{\lambda}, \lambda$ near $\bar{\lambda}$, there is an $r=r(\lambda)$ such that $v(r, \lambda)=0$ and $w(r, \lambda)>-1$. This violates the definition of $\bar{\lambda}$. Similarly, if $\bar{w}>0$, we can reduce this case to the previous one. Finally, the case where $\bar{w}=0$ is dealt with by extending our solution into the complex plane and using the fact that the pair of functions $(w(r), A(r))=\left(0,1+1 / r^{2}-c / r\right)$ is always a solution of $(2 \mathrm{a})$ and $(2 \mathrm{~b})$.

\section{REFERENCES}

1. R. Alder, M. Bazin and M. Schiffer, Introduction to general relativity, 2nd ed., McGrawHill, New York (1975).

2. R. Bartnik and J. McKinnon, Particlelike solutions of the Einstein-Yang Mills equations, Phys. Rev. Lett. 61 (1988), 141-144.

3. S. Soleman, In New Phenomena in Subnuclear Physics (A. Zichichi, ed.), Plenum, New York 1975.

4. S. Deser, Absence of static solutions in source-free Yang-Mills theory, Phys. Lett. B 64 (1976), 463-465.

(J. Smoller and A. Wasserman) Department of Mathematics, University of Michigan, Ann Arbor, Michigan 48109-1003

E-mail address: Joel_Smoller@ub.cc.umich.edu

E-mail address : Arthur_Wasserman@um.cc.umich.edu

(B. Mcleod) Department of Mathematics, University of Pittsburg, Pittsburgh, Pennsylvania 15260

(S. T. Yau) Department of Mathematics, Harvard University, Cambridge, MasSACHUSETTS 02198

E-mail address: styau@math.nthu.edu.tw 